\title{
Contaminación de suelos agrícolas por metales pesados, zona minera El Alacrán, Córdoba-Colombia
}

\author{
Crop soils pollution by heavy metals, the Alacran mining area, \\ Córdoba-Colombia
}

\author{
Zoraya Martínez ${ }^{*}$, María S. González², Jessica Paternina² y Mónica Cantero³ \\ Recibido para publicación: Septiembre 19 de 2016 - Aceptado para publicación: Mayo 18 de 2017
}

\begin{abstract}
RESUMEN
En la zona minera El Alacrán, municipio de Puerto Libertador, departamento de Córdoba, Colombia, la degradación de los recursos naturales como consecuencia de la explotación minera informal de oro es evidente; sin embargo, en la zona el nivel de conocimiento de la problemática relacionada con la explotación y contaminación de metales pesados en suelos agrícolas es deficiente considerando los graves impactos ambientales que viene ocasionando. El objetivo del estudio fue evaluar la contaminación por mercurio, hierro y cobre en suelos agrícolas, y su relación con algunas características químicas del suelo mediante un análisis de tipo multivariado. El área de estudio comprendió un lote de 1,4 ha ubicado a cinco kilómetros aguas abajo del vertimiento de la mina El Alacrán sobre la quebrada Valdéz. Se extrajeron 25 muestras de suelo distribuidas entre zonas eriales y cultivadas, con una densidad de 17,8 muestras/ha. Los resultados mostraron concentraciones excesivas para hierro y cobre totales, y bajas para el mercurio, indicando contaminación por cobre en los suelos agrícolas con valores de TCu que superaron los máximos permisibles en todas las normas internacionales; a diferencia, los valores de mercurio no indicaron polución. Las propiedades químicas de $\mathrm{pH}, \mathrm{MO}$ y $\mathrm{CICe}$ controlan las concentraciones de los metales $\mathrm{Cu}$ y $\mathrm{Fe}$ en el suelo, tanto para las formas totales como para las disponibles. No se econcontraron evidencias estadísticas que permitieran identificar diferentes poblaciones de suelos en relación a las propiedades químicas y metales pesados evaluados.
\end{abstract}

Palabras clave: Contaminación por cobre, mercurio, suelos agrícolas, análisis multivariado.

\begin{abstract}
In El Alacrán mining zone, Puerto Libertador town (Córdoba, Colombia), is evident the natural resources degradation as a result of informal gold mining; however, in this area, the knowledge about problems related to crop soil pollution by heavy metals exploitation is deficient, considering the serious environmental impacts that it has been causing. The aim of this research was to evaluate mercury, iron and copper pollution in crop soils, and its relationship with some soil chemical characteristics through multivariate analysis. The study area was 1.4 ha, which were located five kilometers downstream of the Alacrán mine dump in the Valdéz stream. 25 soil samples were collected between uncultivated and cultivated areas, with a density of 17.8 sample / ha. The results showed excessive total concentrations for iron and copper, and low for mercury, indicating copper contamination in agricultural soils with $\mathrm{TCu}$ values that exceeded the maximum permissible in all international standards; In contrast, mercury values did not indicate pollution. The chemical properties of $\mathrm{pH}, \mathrm{MO}$ and $\mathrm{CICe}$ control the concentrations of $\mathrm{Cu}$ and Fe metals in the soil, both for total and available forms. No statistical evidence was found to identify different soil populations in relation to the chemical properties and heavy metals evaluated.
\end{abstract}

Keywords: Pollution by copper, mercury, agricultural soil, multivariate analysis.

\footnotetext{
${ }_{1 *}$ M.Sc. Profesor asociado. Programa Ingeniería Ambiental. Universidad de Córdoba, Montería, Colombia. Cra 6No.76-103. Laboratorio Integrado Ingeniería Ambiental, +573006545782, zymartinezlara@unicordoba.edu.co Córdoba - Colombia

${ }^{2}$ Estudiantes. Programa de Ingeniería Ambiental. Universidad de Córdoba. Córdoba - Colombia

${ }^{3}$ M.Sc. Profesor. Universidad de Córdoba. Montería. Colombia.
} 


\section{INTRODUCCIÓN}

A nivel mundial las actividades mineras son consideradas como una de las principales fuentes de deterioro de la calidad de los recursos naturales por los impactos negativos generados al ambiente (Stankovic y Stankovic 2013). El principal problema son los relaves mineros que se extienden sobre grandes áreas, los cuales con frecuencia son abandonados con poco o ningún proceso de remediación y ocasionan la migración de metales pesados a los ambientes cercanos, contribuyendo tanto a la contaminación del suelo como a la destrucción del paisaje (Oyarzun et al. 2011; Jordanova et al. 2013; Stankovic y Stankovic 2013).

Los metales pesados guardan una relación directa con los riesgos por contaminación de los suelos, toxicidad en las plantas y los efectos negativos sobre la calidad de los recursos naturales y el ambiente, peligros dependientes de diversos aspectos como son la toxicidad específica del metal, bioacumulación, persistencia y no biodegradabilidad (Oyarzun et al. 2011; Wang et al. 2015).

El municipio de Puerto Libertador, departamento de Córdoba, Colombia, se ha caracterizado por un intenso uso agrícola principalmente en cultivos de arroz y maíz; sin embargo, actualmente la minería es la tercera actividad económica más importante de la zona urbana y la segunda a nivel rural, generando empleos formales e informales en las explotaciones de carbón, níquel, oro y cobre existentes (Alcaldía de Puerto Libertador 2012).

Las explotaciones de oro aluvial en el municipio se dan hacia las riberas y cauces de los ríos San Pedro y San Jorge, y el oro de veta es explotado de manera intensiva en la mina El Alacrán (Alcaldía de Puerto Libertador 2012).

Los sedimentos y lixiviados generados en la mina son vertidos en la quebrada Valdéz sin tratamiento alguno, depositandóse sobre la Ilanura aluvial, y aunque las actividades agrícolas han sido predominantes, se observan zonas eriales que compiten en número y extensión con pastos, indicando una posible degradación del suelo.

La informalidad en la minería de oro está fuertemente ligada a la degradación ambiental, ya que es la principal fuente de polución por mercurio global (Sippl 2015). Este tipo de actividad, según la Organización Internacional del Trabajo (1999) ocupa cerca del 80\% de la Minería Artesanal y a Pequeña Escala (MAPE) en el mundo, y en Colombia la informalidad alcanzó un $87 \%$ de las Unidades de Producción Minera (UPM) auríferas en el año 2011, superando la media del sector minero en Colombia de 63\% (Goñi et al. 2014).

Los estudios de los daños al ambiente debidos a las explotaciones mineras son numerosos y se relacionan con la modificación de las propiedades físicas, químicas y biológicas de los recursos naturales, y la afectación a los ecosistemas y cadenas tróficas (Liu et al. 2013).

En el suelo el mayor peligro reside en su acumulación por las plantas y la transferencia a los animales incluido el hombre (Liu et al. 2013). En general, la distribución de metales pesados en los suelos es un fenómeno complejo que se ve influenciada por factores como el potencial redox, el pH, el contenido de materia orgánica, la capacidad de intercambio catiónico, el nivel de las aguas subterráneas y sus fluctuaciones, entre otros (Lee et al. 2001; Galán y Romero 2008; Jordanova et al. 2013). En las plantas la fitotoxicidad genera especialmente reducción del crecimiento radicular, de la biomasa y la transpiración, clorosis y necrosis en hojas, así como síntomas de senescencia y abscisión (Vázquez et al. 1989; Barceló y Poschenrieder 1992).

A pesar de la escasa información científica sobre la magnitud de la situación en la zona 
Marrugo (2006; 2016) en la mina El Alacrán ha reportado que las concentraciones de mercurio en sedimentos, suelos y plantas, varían desde bajas en suelos a altas en sedimentos; por otra parte la Alcaldía de Puerto Libertador (2012) relaciona algunos problemas ambientales como son el incremento en la turbiedad de ríos y quebradas, la disminución de oxígeno disuelto, la erosión, el daño en suelos de aptitud agrícola y ganadera. Por lo anterior es pertinente, el conocimiento sobre la degradación del suelo aledaño a la mina, en relación con los niveles de polución por diversos metales pesados.

El presente artículo permitió investigar el nivel de contaminación por los metales pesados $\mathrm{Cu}$, Fe y $\mathrm{Hg}$ y la relación con algunas propiedades químicas de los suelos de uso agrícola ubicados en un sector piloto dentro del área de influencia del vertimiento de la mina El Alacrán.

\section{MATERIALES Y MÉTODOS}

El municipio de Puerto Libertador se ubica al sur del departamento de Córdoba, en la región noroccidental de Colombia.

El área de estudio se ubicó en la hacienda La Trilla a $3 \mathrm{~km}$ al noroeste del vertimiento de la mina el Alacrán sobre la quebrada Valdéz, en el municipio de Puerto Libertador (Figura 1).
La quebrada Valdéz es un tributario del río San Jorge, en la cuenca del río Magdalena. Geomorfológicamente el área de estudio es una terraza baja con pendientes inferiores al $3 \%$, formada por sedimentos aluviales de edad Cuaternario que provee la quebrada Valdéz en sus eventos de inundación. La precipitación promedio anual varía entre 2.000 y 4.000 $\mathrm{mm}$, la temperatura promedio anual entre $24{ }^{\circ} \mathrm{C}$ y $36^{\circ} \mathrm{C}$, y se presenta una relación de evapotranspiración (ETP) entre 0,5 y 1,0; la zona de vida pertenece a bosque húmedo tropical (Alcaldía de Puerto Libertador 2005; IGAC 2007). El área de estudio fue seleccionada según los siguientes criterios: i) ubicación en un radio máximo de $5 \mathrm{~km}$ alrededor de la mina, ii) homogeneidad fisiográfica (geomorfología, clima, geología, topografía), iii) uso actual agrícola con evidencias de sectores degradados (zonas sin vegetación), y, iv) sin evidencias de explotación de oro aluvial.

Se georreferenciaron y muestrearon 25 pozos de observación en enero de 2015, de conformidad con un diseño de red rígida, espaciados cada 30 metros; 10 puntos en áreas degradadas (desnudas) y 15 repeticiones en áreas no degradadas o de cultivo (Figura 1). La descripción y toma de muestras en cada pozo se realizó acorde a los criterios de la USDA (2010) y el IGAC (2007). Los parámetros analizados

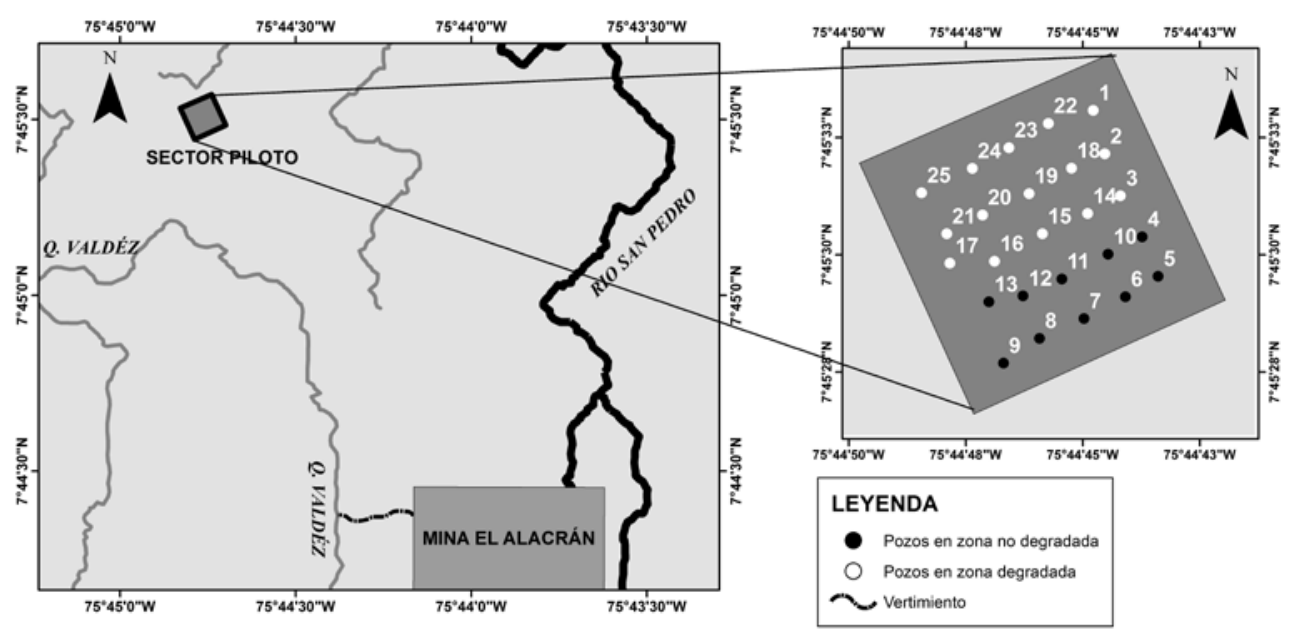

Figura 1. Localización del sector de estudio y área de muestreo. 
fueron $\mathrm{pH}, \mathrm{MO}, \mathrm{CIC}$, macronutrientes $(\mathrm{S}, \mathrm{P}, \mathrm{Ca}$, $\mathrm{Mg}, \mathrm{K}$ y $\mathrm{Na})$, micronutrientes disponibles $(\mathrm{Cu}$ dis, Fe dis, Zn, Mn y B), aluminio intercambiable $\left(\mathrm{Al}^{3+}+\mathrm{H}^{+}\right)$, y concentraciones totales de metales pesados $\mathrm{Hg}$, Cu y Fe (THg, TCu y TFe).

Para el análisis de las propiedades químicas de $\mathrm{pH}$, materia orgánica, $\mathrm{CIC}$, macronutrientes $(\mathrm{S}$, $\mathrm{P}, \mathrm{Ca}, \mathrm{Mg}$, $\mathrm{K}$ y $\mathrm{Na})$, micronutrientes $(\mathrm{Cu}, \mathrm{Fe}, \mathrm{Zn}$, $\mathrm{Mn}$ y B) y aluminio intercambiable se tomó una muestra compuesta de aproximadamente un kilogramo de suelo.

Los métodos de laboratorio usados en la cuantificación de estos parámetros fueron: elementos menores (Cu, Fe, Zn, Mn y B), espectrofotometría de absorción atómica; $\mathrm{pH}$, potenciométrico; \%MO, Walkey-Black; S, espectroscopia UV-VIS (Monofosfato de calcio 0,008M); P, espectroscopia UV-VIS (Bray II modificado); Ca y $\mathrm{Mg}$, espectrofotometría de absorción atómica; $\mathrm{K}$ y $\mathrm{Na}$, espectrofotometría de emisión atómica; $\mathrm{Al}^{++} \mathrm{H}^{+}$, titulación volumétrica; y $\mathrm{CICe}$ con suma de cationes. Para las concentraciones totales de $\mathrm{Cu}, \mathrm{Hg}$ y $\mathrm{Fe}$, se extrajo una muestra de los primeros $15 \mathrm{~cm}$ de suelo y se cuantificaron con el método de espectrofotometría de absorción atómica.

Los datos se analizaron mediante estadística descriptiva y métodos multivariados.

Los estadísticos descriptivos permitieron analizar la variabilidad y fuente de los datos, y el análisis de varianza se implementó mediante la prueba no paramétrica de Kruskal Wallis, con el propósito de establecer la existencia de poblaciones de suelos diferentes acordes a las características químicas y el contenido de metales pesados en el suelo.

Las relaciones entre las variables investigadas se identificaron a través de la correlación ordinal de Spearman y un análisis por componentes principales (ACP), último que acorde a lo propuesto por Mezzadri y Saccani (1989), pretende facilitar la reducción e interpretación de datos mediante la identificación de nuevos componentes síntesis del sistema, con base en la asociación de las variables originales. Los datos fueron procesados en los programas SAS 9.1.3 y Statgraphics XVII-X6.

\section{RESULTADOS Y DISCUSIÓN}

Los suelos fueron clasificados como Inceptisoles con secuencias de baja evolución tipo Ap-Apg/ AB /BC-CB caracterizados por presentar un epipedón ócrico suprayaciendo un endopedón cámbico, donde los rasgos redoximórficos como moteos fueron frecuentes en el $52 \%$ de los perfiles. El horizonte Ap presentó matices 7,5 YR a 10YR, con estructura en bloques angulares a subangulares de débil a moderado desarrollo, y consistencia moderadamente plástica.

El horizonte $A B$ con espesores entre 15 y 22 cm mostró predominio de matices 7,5 YR con estructura de bloques subangulares a angulares de moderado a débil desarrollo y consistencia friable y moderadamente plástica. El horizonte inferior (BC ó CB) presentó estructura de bloques subangulares débilmente desarrollados. Fueron comunes los procesos de hidromorfismo con niveles freáticos muy superfciciales ubicados a profundidades de 20 y 40 centimetros, y las granulometrías dominadas por las clases texturales franco arcilloso (FAr).

Los suelos con baja evolución son comunes en toda la región Caribe de Colombia, ocupando un $62 \%$ de la superficie, según lo reportado por Malagón (2003); específicamente en Córdoba, los Inceptisoles han sido reportados por Combatt et al. (2012) en zonas aluviales de los municipios de Valencia, Tierralta, San Bernardo del Viento y Moñitos.

Las características químicas y la estadística descriptiva de los suelos se presentan en la tabla 1. 
Los valores de $\mathrm{pH}$ fluctuaron entre 3,99 y 5,09, con promedio de 4,76, clasificados como ácidos extremos hasta ácidos muy fuertes (NRCS 1988). De igual forma Hue et al. (2014) señalan que al norte de la provincia de Hunan, la reacción de suelo en antrosoles estágnicos, presentó valores que variaron entre 3,8 a 5,8. Cuando prevalecen $\mathrm{pH}$ bajos en el suelo, los micronutrientes boro, cobre y zinc pueden presentar promedios altos (Prat 1965), incluso llegar a ser tóxicos debido a la alta solubilidad que manifiestan, esta situación, tiende a reducir la capacidad de la planta para absorber fósforo, calcio, magnesio y molibdeno (FAO 2000).

El contenido moderado a excesivo de aluminio intercambiable $\left(0,16-2,73 \mathrm{cmol}^{+} \mathrm{kg}^{-1}\right)$, es coherente con los valores de $\mathrm{pH}$ observados. Los contenidos promedio de materia orgánica y $\mathrm{ClCe}$ fueron moderados, indicando cierta inhibición en la mineralización de la materia orgánica, posiblemente asociado a los procesos hidromórficos del sistema (Tabla 1).

Promedios altos para los micronutrientes $\mathrm{Cu}$, $\mathrm{Fe}, \mathrm{Zn}$ y $\mathrm{Mn}$ fueron encontrados, al igual que para $\mathrm{TCu}$ y $\mathrm{TFe}$, las concentraciones de $\mathrm{THg}$ fueron bajas (Tabla 1).

Tabla 1. Estadística descriptiva de las variables químicas y metales pesados en los suelos del sector piloto.

\begin{tabular}{|c|c|c|c|c|c|}
\hline Variable & Media & $D E$ & $C V$ & Mínimo & Máximo \\
\hline $\mathrm{pH}$ & 4,76 & 0,28 & 5,91 & 3,99 & 5,09 \\
\hline MO (\%) & 2,09 & 0,70 & 33,36 & 1,13 & 3,46 \\
\hline $\mathrm{S}$ & 28,51 & 22,86 & 80,18 & 7,70 & 77,30 \\
\hline$P$ & 3,16 & 2,97 & 93,70 & 0,50 & 12,30 \\
\hline $\mathrm{Ca}$ & 10,37 & 2,89 & 27,90 & 6,10 & 16,30 \\
\hline$M g$ & 8,23 & 2,86 & 34,77 & 4,60 & 14,50 \\
\hline K & 0,12 & 0,06 & 48,66 & 0,07 & 0,30 \\
\hline $\mathrm{Na}$ & 0,20 & 0,07 & 37,08 & 0,09 & 0,38 \\
\hline $\mathrm{Al}+\mathrm{H}$ & 0,97 & 0,76 & 78,95 & 0,16 & 2,73 \\
\hline $\mathrm{ClCe}$ & 19,85 & 4,83 & 24,35 & 13,10 & 31,20 \\
\hline Cu_d & 83,16 & 91,98 & 110,61 & 19,40 & 378,00 \\
\hline Fe_d & 74,48 & 79,66 & 106,96 & 20,60 & 310,90 \\
\hline $\mathrm{Zn}$ & 5,13 & 1,36 & 26,41 & 3,20 & 7,30 \\
\hline Mn & 178,51 & 107,70 & 60,33 & 76,90 & 492,60 \\
\hline B & 0,28 & 0,12 & 41,99 & 0,12 & 0,50 \\
\hline $\mathrm{THg}$ & 0,11 & 0,05 & 47,61 & 0,06 & 0,26 \\
\hline $\mathrm{TCu}$ & 428,60 & 354,31 & 82,67 & 59,84 & 1453,58 \\
\hline TFe & 67988,00 & 23082,00 & 33,95 & 29052,50 & 107365 \\
\hline
\end{tabular}

DE desviación estándar. CV coeficiente de variación (\%). Unidades $\mathrm{pH}$ : potenciométrico; $\mathrm{MO}$ \% \% $\mathrm{Ca}, \mathrm{Mg}, \mathrm{K}, \mathrm{Na}, \mathrm{ClCe}$ y $\mathrm{Al}^{+} \mathrm{H}: \mathrm{cmol}^{+} \mathrm{kg}^{-1}$. Las restantes concentraciones están reportadas en mg kg-1 de peso seco.
Los datos obtenidos para TFe y THg presentaron menor variabilidad que los de TCu. La polución por metales pesados tiende a generar acidez en los suelos (Sahuquillo et al. 2003; Prieto et al. 2009), proceso ampliamente confirmado en áreas mineras y sus vecindades, como lo reporta Yin et al. (2016) en la provincia de Guandong en China, donde los suelos en cercanías a explotaciones mineras mostraron $\mathrm{pH}$ entre 2,4 a 6,7.

Se observó una alta variabilidad para los parámetros Cu_d, Fe_d, P, TCu y S, presentando coeficientes de variación mayores a $80 \%$; en contraste, las variables menos dispersas fueron $\mathrm{pH}, \mathrm{MO}, \mathrm{Zn}, \mathrm{Ca}, \mathrm{Mg}$ y TFe, con coeficientes de variación menores al 35\% (Tabla 1).

Los metales pesados mostraron concentraciones medias, mínimas y máximas según el patrón $\mathrm{Fe}>\mathrm{Cu}>\mathrm{Hg}$ (Tabla 1), exhibiendo TCu un coeficiente de variación cercano al 100\% y concentraciones anormalmente altas. La variabilidad observada en los datos de ciertas propiedades puede obedecer tanto a la variabilidad espacial natural en los suelos, como al aporte proveniente de fuentes diferentes a la natural.

En el caso del TCu, las concentraciones anormalmente altas reportadas en este estudio $\left(59,84-1453,58 \mathrm{mg} \mathrm{kg}^{-1}\right)$, en comparación con los valores medios de cobre en rocas y suelos de la corteza terrestre de 2 - 100 mg $\mathrm{kg}^{-1}$ (Oyarzun et al. 2011), sugieren una fuente que difiere de la natural y es más cercana a un origen antropogénico (Yacoub et al. 2012; Diatta et al. 2015).

El Ministerio de Agricultura de Chile (2005) al relacionar los niveles máximos de metales pesados en suelos para diversas normas internacionales, indicaqueexistecontaminación severa por cobre con necesidad de intervención, cuando se alcanzan concentraciones de 190 $\mathrm{mg} \mathrm{kg}{ }^{-1}$ en la norma holandesa, $100 \mathrm{mg} \mathrm{kg-1}$ en la ley federal alemana, y $150 \mathrm{mg} \mathrm{kg}^{-1}$ para 
Chile zona norte. Aunque pueden existir suelos naturales geoquímicamente anómalos en las franjas metalogénicas donde son abundantes ciertos minerales ricos en metales, en Chile valores de $\mathrm{Cu}>200 \mathrm{mg} \mathrm{kg}^{-1}$, son considerados anómalos excesivos, valores que para Diatta et al. (2015) pueden relacionarse con actividades mineras que son unas de las mayores fuentes de contaminación por cobre a nivel mundial.

Para el caso del hierro, valores altos se han encontrado cerca de la zona minera de la provincia de Guangdong en China, reportando para suelos naturales entre 78 y $97 \mathrm{~g} \mathrm{~kg}^{-1}$, y para suelos de residuos mineros entre 257 a $438 \mathrm{~g}$ $\mathrm{kg}^{-1}$ (Yin et al. 2016); sin embargo, los altos valores medios hallados en esta investigación de $67971 \mathrm{mg} \mathrm{kg}^{-1}$, se relacionan con lo reportado por Belmonte et al. (2010) en suelos agrícolas cercanos a una mina con ricos yacimientos de hierro en el sureste de España.

Las muestras de suelo para el análisis de las propiedades físicas, químicas y el contenido de metales pesados, fueron seleccionadas con base en el criterio de ausencia o presencia de vegetación como respuesta al estado de degradación del suelo. Los valores obtenidos de esta caracterización fueron sometidos a la prueba no parámetrica de Kruskal Wallis $(\mathrm{p}<0,05)$ para identificar el número de poblaciones de procedencia. Los resultados indicaron que la probabilidad asociada a cada una de las variables superó en todos los casos el valor de 0,05 establecido como límite, por lo que el estadístico de la prueba no presenta ningún nivel de significancia para los parámetros analizados y se rechaza la hipótesis de la existencia de dos áreas con características o estados de degradación diferentes.

Se comprueba por tanto la existencia de una sola población de datos debido a la similitud en la varianza de los parámetros, aspecto que permite afirmar la existencia de suelos con características químicas y niveles de degradación similares; resultados equivalentes fueron hallados por Hu et al. (2014) en suelos provenientes de cuatro campos de arroz afectados por descargas de aguas residuales mineras al norte de la provincia de Hunan, los cuales se clasificaron como Antrosoles estágnicos por sus similitudes en las propiedades físicas y químicas evaluadas.

Las relaciones entre las propiedades químicas del suelo, y de estas con los metales pesados fueron analizadas mediante correlación de Spearman y Análisis por Componentes Principales (ACP).

Basados en los resultados de la correlación ordinal de Spearman presentados en la tabla

Tabla 2. Correlaciones ordinales de Spearman entre las propiedades quimicas y los metales pesados en suelos cercanos a la mina El Alacrán.

\begin{tabular}{|c|c|c|c|c|c|c|c|c|c|c|c|c|c|c|c|c|c|c|}
\hline & $\mathrm{pH}$ & $\mathrm{MO}$ & S & $\mathrm{P}$ & $\mathrm{Ca}$ & $\mathrm{Mg}$ & K & $\mathrm{Na}$ & $\mathrm{Al}+\mathrm{H}$ & $\mathrm{ClC}$ & Cu_d & Fe_d & $\mathrm{Zn}$ & $\mathrm{Mn}$ & B & Hg_t & $\mathrm{Cu}$ _t & Fe_t \\
\hline $\mathrm{pH}$ & 1 & $-0,02$ & $-0,46^{* *}$ & $-0,14$ & $0,46^{* *}$ & 0,34 & $-0,31$ & $0,44^{* *}$ & $-0,67^{* *}$ & 0,38 & $-0,32$ & $-0,14$ & $-0,05$ & $-0,30$ & $-0,66^{* *}$ & 0,17 & $-0,16$ & $-0,12$ \\
\hline $\mathrm{MO}$ & & 1 & 0,18 & $0,42^{* *}$ & 0,05 & $-0,11$ & 0,32 & $-0,09$ & 0,22 & 0,05 & 0,01 & $0,43^{* *}$ & 0,35 & 0,34 & $-0,01$ & 0,03 & $-0,53^{* *}$ & $-0,43^{* *}$ \\
\hline$S$ & & & 1 & 0,19 & $-0,03$ & $-0,34$ & 0,40 & $-0,59 * *$ & 0,36 & $-0,16$ & 0,35 & 0,09 & 0,04 & $0,41^{* *}$ & $0,52 * *$ & $-0,13$ & $-0,03$ & $-0,33$ \\
\hline P & & & & 1 & 0,21 & $-0,31$ & 0,10 & $-0,05$ & 0,25 & 0,07 & $-0,16$ & 0,16 & 0,03 & 0,28 & 0,03 & $-0,20$ & $-0,11$ & $-0,25$ \\
\hline $\mathrm{Ca}$ & & & & & 1 & $0,45^{* *}$ & $-0,15$ & 0,29 & $-0,63^{* *}$ & $0,89^{* *}$ & $-0,17$ & $-0,47^{* *}$ & $-0,23$ & $-0,31$ & $-0,35$ & $-0,14$ & $-0,21$ & $-0,26$ \\
\hline $\mathrm{Mg}$ & & & & & & 1 & $-0,07$ & $0,42 * *$ & $-0,44^{* *}$ & $0,72^{* *}$ & $-0,03$ & $-0,26$ & $-0,28$ & $-0,80^{* *}$ & $-0,10$ & $-0,26$ & $-0,14$ & 0,16 \\
\hline$K^{\circ}$ & & & & & & & 1 & $-0,62^{* *}$ & 0,36 & $-0,13$ & $0,81^{* *}$ & $0,44^{* *}$ & $0,55^{* *}$ & 0,30 & $0,63^{* *}$ & 0,10 & $-0,30$ & $-0,15$ \\
\hline $\mathrm{Na}$ & & & & & & & & 1 & $-0,36$ & $0,43^{* *}$ & $-0,56^{* *}$ & $-0,16$ & $-0,51^{* *}$ & $-0,38$ & $-0,61^{* *}$ & $-0,21$ & $-0,08$ & 0,15 \\
\hline $\mathrm{Al}+\mathrm{H}$ & & & & & & & & & 1 & $-0,55^{* *}$ & 0,14 & $0,62 * *$ & 0,20 & $0,49 * *$ & $0,53^{* *}$ & $-0,14$ & $-0,08$ & $-0,10$ \\
\hline $\mathrm{ClC}$ & & & & & & & & & & 1 & $-0,15$ & $-0,41 * *$ & $-0,33$ & $-0,52 * *$ & $-0,32$ & $-0,16$ & $-0,22$ & $-0,15$ \\
\hline Cu_d & & & & & & & & & & & 1 & 0,15 & 0,32 & 0,15 & $0,70^{* *}$ & 0,17 & 0,01 & 0,02 \\
\hline Fe_d & & & & & & & & & & & & 1 & $0,45^{* *}$ & $0,43^{* *}$ & 0,27 & 0,18 & $-0,20$ & $-0,04$ \\
\hline $\mathrm{Zn}$ & & & & & & & & & & & & & 1 & $0,47^{* *}$ & 0,27 & 0,31 & $-0,21$ & $-0,16$ \\
\hline $\mathrm{Mn}$ & & & & & & & & & & & & & & 1 & 0,18 & 0,29 & $-0,21$ & $-0,42^{* *}$ \\
\hline B & & & & & & & & & & & & & & & 1 & $-0,19$ & 0,09 & 0,01 \\
\hline $\mathrm{Hg} \mathrm{t}_{\mathrm{H}}$ & & & & & & & & & & & & & & & & 1 & 0,24 & 0,04 \\
\hline $\mathrm{Cu}$-t & & & & & & & & & & & & & & & & & 1 & $0,51^{* *}$ \\
\hline Fe_t & & & & & & & & & & & & & & & & & & 1 \\
\hline
\end{tabular}


2, las parejas con alta correlación negativa $(\mathrm{p}<0,05)$ fueron: $\mathrm{pH}-\mathrm{Al}^{3+}+\mathrm{H}^{+}, \mathrm{pH}-\mathrm{S}, \mathrm{MO}-$ $\mathrm{TCu}, \mathrm{MO}-\mathrm{TFe}, \mathrm{Al}^{3+}+\mathrm{H}^{+}-\mathrm{CICe}$ y CICe $-\mathrm{Fe} \_d ; \mathrm{y}$ las parejas con alta correlación positiva fueron $\mathrm{MO}-\mathrm{Fe} \_\mathrm{d}, \mathrm{Al}^{3+}+\mathrm{H}^{+}-\mathrm{Fe} \_\mathrm{d}$ y TCu-TFe.

Luego de la selección de las variables con fuerte correlación, y para garantizar la idoneidad en la aplicación de ACP, se aplicó el estadístico de Kaiser-Meyer-Olkin (KMO) que garantiza la factorabilidad en la población de datos. KMO arrojó un valor de 0,63 por encima del límite de 0,6, indicando que la factorización era posible y podía proveer información sobre los componentes principales. De conformidad con los resultados de ACP se seleccionaron los tres primeros componentes por mostrar eigenvalores $>1$; a cada variable en los componentes se le atribuyó un valor $\lambda$ que representaba la cantidad de información o el peso al interior del componente, en este caso, se analizaron las variables con pesos superiores a $\lambda>75 \%(0,75)$.

La tabla 3 muestra la distribución por pesos de la variables que componen cada uno de los componentes principales seleccionados, los cuales en conjunto explicaron el $81 \%$ de la varianza del sistema, y la figura 2 describe la distribución espacial de los componentes en el ACP.

El primer componente explicó el 38,87\% de la varianza total y se interpretó a partir de la dinámica de la $\mathrm{MO}$ que controla indirectamente la concentración de TCu y TFe, a diferencia, los incrementos de la materia orgánica favorecen

Tabla 3. Estructura de los componentes principales y pesos de las variables que los conforman.

\begin{tabular}{lccc}
\hline Variable & Componente 1 & Componente 2 & Componente 3 \\
\hline $\mathrm{Al}^{3+}+\mathrm{H}^{+}$ & 0,27 & 0,54 & 0,68 \\
$\mathrm{CICe}$ & 0,26 & $-0,91$ & $-0,07$ \\
$\mathrm{TCu}$ & $-0,76$ & $-0,02$ & 0,39 \\
$\mathrm{Cu} d$ & $-0,10$ & $-0,08$ & 0,92 \\
$\mathrm{TFe}$ & $-0,82$ & 0,11 & $-0,01$ \\
$\mathrm{Fe} \_d$ & 0,59 & 0,72 & 0,11 \\
$\mathrm{Mn}$ & 0,60 & 0,72 & 0,16 \\
$\mathrm{MO}$ & 0,79 & 0,26 & $-0,01$ \\
$\mathrm{pH}$ & 0,15 & $-0,22$ & $-0,88$ \\
\hline
\end{tabular}

la disponibilidad de los metales Mn y Fe_d. Se observa en este componente la alta correlación positiva entre TFe y TCu (Figura 2).

El segundo componente recogió el 29,22\% de la varianza y estuvo controlado por la $\mathrm{CICe}$, reflejando la negativa relación de la $\mathrm{CICe}$ en la disponibilidad de los micronutrientes Fe y Mn; se observa una alta correlación positiva entre Fe_d y Mn (Figura 2).
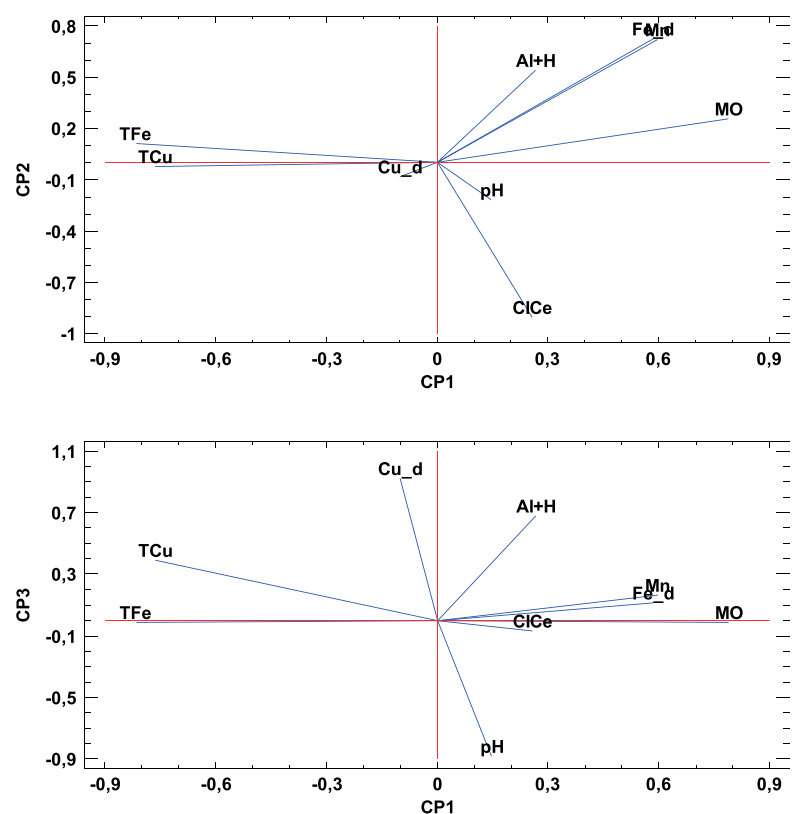

Figura 2. Distribución bidimensional de las variables en el análisis de componentes principales.

El tercer componente presentó el 12,96\% de la varianza, mostrando la incidencia inversa del $\mathrm{pH}$ sobre la disponibilidad del cobre y la concentración de $\mathrm{Al}^{3+}+\mathrm{H}^{+}$(Figura 2).

Al respecto Zeng et al. (2011) señalan que las propiedades relacionadas con la absorción y desorción de metales pesados en el suelo incluyen el $\mathrm{pH}$, el contenido de materia orgánica, la capacidad de intercambio catiónico (CEC), el estado de oxidación-reducción (Eh), el contenido de minerales arcillosos, carbonato de calcio y óxidos de hierro y manganeso. 
La reacción ácida del suelo puede ser atribuida al incremento de aluminio intercambiable en el medio, el régimen de precipitaciones y el deficiente drenaje del sector de estudio (Bohn et al. 1993; Jaramillo 2002; Chandrasekaran y Ravisankar 2015).

Es clara la relación directa de los medios ácidos y la disponibilidad de los micronutrientes, aspecto discutido ampliamente en la literatura, donde el $\mathrm{pH}$ del suelo es el principal factor que induce la movilidad y la biodisponibilidad de metales pesados en las plantas (Cheng 2003; Adamczyk et al. 2015). En general se establece que a $\mathrm{pH}$ bajos existe una mayor movilidad y absorción de los metales, y que en presencia de altas alcalinidades las plantas no son propensas a absorber ciertos metales (Violantel et al. 2010; Zeng et al. 2011; Adamczyk-Szabela et al. 2015). Adicionalmente, se podría relacionar el incremento de $\mathrm{Al}^{3+}$ con la inhibición en el crecimiento de las plantas (Roca et al. 2007; Chandrasekaran y Ravisankar 2015).

La materia orgánica es además del $\mathrm{pH}$, otra de las propiedades que inciden de forma importante en la disponibilidad de los metales pesados en el suelo, ya que presenta la posibilidad de retenerlos de forma intercambiable y aporta a la solución del suelo productos que favorecen la formación de quelatos y la disponibilidad de los metales (McCauley et al. 2009; Zeng et al. 2011).

La capacidad de la materia orgánica para fijar fuertemente hierro y cobre en las formas disponibles, a través de los grupos $\mathrm{OH}, \mathrm{C}=\mathrm{O}$ y $\mathrm{CO}-\mathrm{OH}$ de los ácidos húmicos y fúlvicos incide en la formación de complejos organometálicos estables (Barral et al. 1997; Seoánez 1999; Galán y Romero 2008), que disminuyen las concentraciones totales de los metales pesados (Adamczyk et al. 2015). Lo anterior se refleja en las relaciones inversas $\mathrm{MO}-\mathrm{TCu}, \mathrm{MO}-\mathrm{TFe}$ del primer componente principal y en los resultados de las correlaciones ordinales.
El comportamiento de la CICe sugiere una fuerte dependencia del $\mathrm{pH}$, ya que las correlaciones negativas con los micronutrientes sugieren una importante disponibilidad de los metales en medios ácidos.

Las concentraciones excesivas TFe y TCu, y su alta correlación positiva sugieren un origen similar, relacionado con fuentes antropogénicas (Li y Feng 2012; Pan et al. 2016). Al respecto, Silva et al. (2014) afirman que las altas correlaciones positivas entre elementos típicos de menas metálicas en sedimentos, son reflejo del impacto de las actividades mineras.

El caso del THg indica un origen diferente al de TFe y TCu, en general este metal no presentó correlación alguna con las propiedades químicas del suelo; lo anterior está relacionado con la paragénesis del sector, abundante en sulfuros de hierro y cobre, donde no existen evidencias de menas de mercurio.

Las características geológicas y bio-climáticas de una región controlan las concentraciones naturales de los elementos químicos presentes en el suelo, aspecto conocido como fondo geoquímico local (FG) o nivel de fondo, el cual representa una situación ideal que permite determinar la contaminación por metales presentes en concentraciones inusualmente elevadas (Diez 2008). Debido a que la legislación colombiana no contempla valores de referencia para contaminación de suelos y que la zona de estudio no reporta estudios de fondo geoquímico, se realizó una primera aproximación del nivel de contaminación para mercurio y cobre mediante la comparación con los valores máximos permisibles de normas internacionales (Tabla 4).

El hierro por ser usualmente considerado un metal pesado no tóxico, no se reporta en los estándares de contaminación para suelos (Micó et al. 2006), por lo que los valores aquí obtenidos se compararon con las medias encontradas en diferentes referentes internacionales. 
Tabla 4. Valores máximos de referencia internacional para determinar contaminación para $\mathrm{Cu}$ y $\mathrm{Hg}$, y concentraciones promedio de Fe en los suelos.

\begin{tabular}{|c|c|c|c|}
\hline Metal & Referencia & Valor & País \\
\hline $\mathrm{Cu}$ & Máximo ${ }^{1}$ & 100 & Austria, Canadá, Polonia \\
\hline $\mathrm{Cu}$ & Máximo ${ }^{1}$ & 50 & $\begin{array}{l}\text { Inglaterra } \\
\text { Int }\end{array}$ \\
\hline $\mathrm{Cu}$ & Máximo² & 60 & Alemania \\
\hline $\mathrm{Hg}$ & Máximo ${ }^{1}$ & 5 & Austria, Polonia \\
\hline $\mathrm{Hg}$ & Máximo ${ }^{1}$ & 0,3 & Canadá \\
\hline $\mathrm{Hg}$ & Máximo ${ }^{1}$ & 2 & Inglaterra \\
\hline $\mathrm{Hg}$ & Máximo² & 12 & Alemania \\
\hline $\mathrm{Fe}$ & Medio $^{3}$ & 66123 & \\
\hline $\mathrm{Fe}$ & Medio $^{4}$ & 50000 & \\
\hline $\mathrm{Fe}$ & Medio $^{4}$ & 40000 & \\
\hline $\mathrm{Fe}$ & Medio $^{5}$ & 25000 & \\
\hline $\mathrm{Fe}$ & Medio $^{6}$ & 19100 & \\
\hline $\mathrm{Fe}$ & Medio $^{7}$ & 14770 & \\
\hline
\end{tabular}

Valores de referencia para metales pesados en $\mathrm{mg} \mathrm{kg}^{-1}$ por país y/o autor. 1 Kabata-Pendias (1984). 2 Ley Federal Alemana, Ordenanza BboSch/1999. 3 Belmonte et al. (2010). 4 Istvan País y Benton Jr. (1997). 5 Cottenie et al. (1979). 6 Williams et al. (1980). 7 Frank et al. (1976).

Las concentraciones de mercurio $(<0,26 \mathrm{mg}$ $\left.\mathrm{kg}^{-1}\right)$ estuvieron por debajo del nivel máximo permisible más restrictivo de $0,3 \mathrm{mg} \mathrm{kg}^{-1}$, perteneciente a la norma canadiense; por lo cual el suelo del área piloto no se encuentra contaminado por mercurio (Tabla 3).

Esto obedece a que parte del metal incluido en la amalgamación puede concentrarse y contaminar de forma local, y su dispersión se limita al área de operación o se volatiliza transformándose al estado gaseoso (Niane et al. 2014), también puede disponerse en lugares aguas arriba cercanos al vertimiento de la quebrada y no en sitios más alejados como la terraza en estudio (Lecce y Pavlowsky 2006).

Marrugo (2006) encontró altas concentraciones de $\mathrm{Hg}\left(>1 \mu \mathrm{g} \mathrm{g}^{-1}\right)$ en estudios realizados sobre la quebrada Valdéz y los sedimentos de la mina El Alacrán, y en 2016 reportó bajas concentraciones relativas de $\mathrm{THg}(0,23$ - 6,32 mg $\mathrm{kg}^{-1}$ ) en suelos de la mina en comparación con otras áreas contaminadas en el mundo (Marrugo et al. 2016).

Los datos del presente estudio indicaron concentraciones de cobre (59,8 - 1453,6 mg $\mathrm{kg}^{-1}$ ) que en el $88 \%$ de los casos superan los niveles máximos permisibles en las normas de Austria, Canadá, Polonia e Inglaterra, y en un
96\% para la norma alemana. El Ministerio de Agricultura de Chile (2005) afirma que aún en las franjas metalogénicas ricas en cobre, el valor máximo de cobre en suelos es de $200 \mathrm{mg} \mathrm{kg}^{-1}$.

Lo anterior define una severa contaminación por cobre en la zona de estudio cuyo origen puede estar directamente relacionado con el vertimiento de residuos que la Mina El Alacrán realiza hacia la quebrada Valdéz.

Esta situación se relaciona con el beneficio del oro en la mina El Alacrán, ya que los minerales de cobre presentes en la paragénesis del depósito no son aprovechados, implicando la eliminación de volúmenes importantes de canga enriquecida en cobre, los cuales viajan por la corriente y son distribuidos en su llanura en épocas de inundación, o por irrigación, dinámicas de transporte y acumulación de contaminantes, procesos que según Diatta et al. (2015) facilitan la polución por las actividades mineras y metalúrgicas.

Los valores medios de hierro en suelos varían en atención a las características geoquímicas de la zona, sin embargo, las concentraciones para todos los puntos analizados superaron los valores promedio propuestos por diferentes autores.

El contenido medio de hierro en el sector piloto de $67.988 \mathrm{mg} \mathrm{kg}^{-1}$, guarda una estrecha relación a lo encontrado por Belmonte et al. (2010) en suelos agrícolas cercanos a una mina con ricos yacimientos de hierro en el sureste de España, depósito constituido principalmente por galena, pirita y óxidos férricos como goethita y hematites.

\section{CONCLUSIONES}

Se observan rasgos de degradación en las áreas de cultivo como zonas eriales, no existe evidencia estadística que las poblaciones de suelos sean diferentes, en consideración a las propiedades químicas o concentraciones de los metales pesados en el sistema. 
La naturaleza geológica del yacimiento está relacionada con minerales ricos en sulfuros de hierro y cobre, la alta correlación positiva entre TFe y TCu, y las altas concentraciones de estos elementos, reflejan el impacto de la minería en el sector. Las anomalías de estos metales tienen relación con fuentes de carácter antropogénico, como la actividad minera El Alacrán.

Una primera aproximación al nivel de contaminación para $\mathrm{Cu}, \mathrm{Hg}$ y $\mathrm{Fe}$ en suelos, mediante la comparación con referentes internacionales, indica que existe polución por cobre por encima de todos los valores máximos permisibles en las normas analizadas, no existe contaminación de $\mathrm{Hg}$ en el suelo, y su origen es diferente al de los metales Fe y $\mathrm{Cu}$.

\section{AGRADECIMIENTOS}

A la Universidad de Córdoba por la financiación del proyecto "Caracterización de suelos afectados por la explotación de oro en un sector piloto de la vereda mina El AlacránPuerto Libertador, Córdoba".

\section{REFERENCIAS}

Adamczyk-Szabela, D., Markiewicz, J. and Wolf, W. 2015. Heavy Metal Uptake by Herbs. IV. Influence of Soil $\mathrm{pH}$ on the Conten $\mathrm{t}$ of Heavy Metals in Valeriana officinalis L. Water Air and Soil Pollution 226(4): 106. https://doi.org/10.1007/ s11270-015-2360-3

Alcaldía del municipio de Puerto Libertador. Plan de Desarrollo municipal. 20122015. http://www.puertolibertador-cordoba.gov.co/apc-aa-files/. [13 junio 2016]

Barceló, J.y Poschenrieder, Ch. 1992. Respuestas de las plantas a la contaminación por metales pesados. Suelo y Planta 2(2): 345-361.
Barral, M., Arias, M. y Diaz, F. 1997. Influencia del hierro y el aluminio en el contenido de materia orgánica en el suelo. Bioloxia 7:137-144.

Belmonte, F., Romero,A., Alonso, F., Moreno J. y Rojo S. 2010. Afección de suelos agrícolas por metales pesados en áreas limítrofes a explotaciones mineras del sureste de España. Papeles de geografía 51-52:45-54.

Bohn, H., McNeal, B. and O'Connor, G. 1993. Química del suelo. Limusa, México. 370p.

Combatt, E., Novoa, R. y Barrera, J. 2012. Caracterización química de macroelementos en suelos cultivados con plátano (Musa AAB Simmonds) en el departamento de Córdoba, Colombia. Acta Agronómica 61(2):166-176.

Chandrasekaran, A. and Ravisankar, R. 2015. Spatial distribution of physicochemical properties and function of heavy metals in soils of Yelagiri hills, Tamilnadu by energy dispersive X-ray florescence spectroscopy (EDXRF) with statistical approach. Spectrochimica Acta Part A: Molecular and Biomolecular Spectroscopy 150:586-601. https://doi. org/10.1016/j.saa.2015.05.083.

Diatta, J., Chudzinska, E., Drobek, L., Wojcicka-Połtorak, A., Markert, B. and Wünschmann, S. 2005. Multiple-Phase Evaluation of Copper. Geochemistry En: Sherameti, I. y Varma, A. (Ed). Heavy Metal Contamination of Soils. doi 10.1007/978-3-31914526-6. [26 Enero 2016]. https://doi. org/10.1007/978-3-319-14526-6.

Diez, J. 2008. Fitocorrección de suelos contaminados con metales pesados: Evaluación de plantas tolerantes y optimización del proceso mediante prácticas agronómicas. Tesis doctoral en Biología, Universidad de Santiago de Compostela, Galicia. 
FAO. 2000. Irrigated wheat. http://www.fao. org/docrep/006/x8234s/x8234s08.htm.

Prieto, J., González., Román, A. y Prieto, F. 2009. Contaminación y fitotoxicidad en plantas por metales pesados provenientes de suelos y agua. Tropical and Subtropical Agroecosystems 10 (1): 29-44.

Galán, A. y Romero, E. 2008. Contaminación de suelos por Metales pesados. Macla Revista de la sociedad española de mineralogía 10. http://www.ehu.eus/sem/ macla_pdf/macla10/Macla10_48.pdf [28 Agosto 2015].

Goñi, E., Sabogal, A. y Asmat, R. 2014. Minería informal aurífera en Colombia. Principales resultados del levantamiento de la línea de base. IBD- FEDESARROLLO. http://www.repository.fedesarrollo.org. co/bitstream/11445/368/3/Mineria\%20 informal \% 20 aurifera $\% 20$ en $\% 20$ Colombia\%20-\%20Informe_linea_base_ mineria_informal\%20-\%20pagina\%20 Fedesarrollo.pdf. [5 Julio 2016]

Hu, X., Jiang, Y., Shua, Y., Hu, X., Liu, L. and Luo, F. 2014. Effects of mining wastewater discharges on heavy metal pollution and soil enzyme activity of the paddy fields. Journal of Geochemical Exploration 147:139-150. https://doi.org/10.1016/j. gexplo.2014.08.001.

IGAC. 2007. Estudio semidetallado de suelos de las áreas potencialmente agrícolas, Bajo Cauca. Imprenta Nacional de Colombia.

Jaramillo, D. 2002. Introducción a la ciencia del suelo. Universidad Nacional de Colombia, Medellín, p415-432.

Jordanova, D., Rao, S., Kotsev, T. y Jordanova, N. 2013. Industrial contamination of alluvial soils near $\mathrm{Fe}-\mathrm{Pb}$ mining site revealed by magnetic and geochemical studies. Geoderma 192:237-248. https://doi. org/10.1016/j.geoderma.2012.07.004.
Lecce, S. and Pavlowsky, R. 2014. Floodplain storage of sediment contaminated by mercury and cooper from historic gold mining at Gold Hill, North Carolina, USA. Geomorphology 206:122-132. https://doi.org/10.1016/j. geomorph.2013.10.004

Lee, C., Chon, H. and Jung, M. 2001. Heavy metal contamination in the vicinity of the Daduk Au-Ag-Pb-Zn mine in Korea. Appl Geochem 16(11-12):1377-1386. https:// doi.org/10.1016/S0883-2927(01)00038-5

Li, X. and Feng, L. 2012. Geostatistical analyses and fractionation of heavy metals in urban soil from industrial district in Weinan, NW China. Environmental Earth Sciences 67(7): 2129-2140. https://doi. org/10.1007/s12665-012-1653-8.

Liu, X., Song,Q., Tang,Y., Li, W., Xu, J., Wu,J., Wang, F. y Brookes, P. 2013. Human health risk assessment of heavy metals in soil-vegetable system: A multi-medium analysis. Science of the Total Environment 463-464C:530-540. Doi: 10.1016/j. scitotenv.2013.06.064

Malagón, D. 2003. Ensayo sobre tipología de suelos colombianos -Énfasis en génesis y aspectos ambientales. Rev. Acad. Colomb. Cienc. 27(104): 319-341.

Marrugo, J. 2006. Impacto ambiental por contaminación con níquel, mercurio y cadmio en aguas, peces y sedimentos en la cuenca del río San Jorge, en el departamento de Córdoba. Centro de investigaciones de la Universidad de Córdoba (CIUC). Montería.

Marrugo, J., Marrugo, S., Pinedo, J., Durango, J. and Diez, S. 2016. Screening of native plant species for phytoremediation potential at a $\mathrm{Hg}$ - contaminated mining site. Science of the Total Environment 542:809-816. https://doi.org/10.1016/j. scitotenv.2015.10.117. 
McCauley, A., Jones, C. and Jacobsen, J., 2009. Soil $\mathrm{pH}$ and Organic Matter. Nutrient management modules 8. Montana State University Extension Service. http:// store.msuextension.org/publications/ agandnaturalresources/4449/4449_8.pdf [13 Julio 2015].

Mezzadri, G. y Saccani, E. 1989. Heavy mineral distribution in late quaternary sediment dispersal in sedimentary basins at active margins. Journal of Sedimentary Petrology 59 (3): 412-422.

Micó, C., Recatalá, L., Peris, M. and Sánchez, J. 2006. Assessing heavy metal sources in agricultural soils of an European Mediterranean area by multivariate analysis. Chemosphere 65 (5):863-872. https://doi.org/10.1016/j. chemosphere.2006.03.016.

Ministerio de Agricultura Chile SAG. 2005. Criterios de calidad de suelos y aguas de riego.http://bibliotecadigital.sag.gob.cl/ documentos/medio_ambiente/criterios_ calidad_suelos_aguas_agricolas/inicio. htm [13 Julio 2015].

Niane, B., Moritz, R., Guedron, S., Malick, P., Rudolf, H., Mall, I. y and Poté, J. 2014. Effect of recent artisanal small-scale gold mining on the contamination of surface river sediment: Case of Gambia River, Kedaugou region. Southeastern Senegal. Journal of geochemical exploration 144 C:217-527. https://doi.org/10.1016/j. gexplo.2014.03.028.

NRCS. 1998. National Soil Survey Handbook. USDA.

Organización Internacional del Trabajo. 1999. Social and Labour Issues in Small-Scale Mines. Report for discussion at the Tripartite Meeting on Social and Labour Issues in Small-scale Mines, ILO, Geneva. http://ilo.org/public/english/dialogue/ sector/techmeet/tmssm99/tmssmr.htm [20 Julio 2015].
Oyarzun, R., Higueras, P. y Lillo, J. 2011. Minería ambiental: Una introducción a los impactos y su remediación. Ediciones GEMM, España, p57-61, p91-123.

Pan, LB., Ma, J., Wang, XL. and Hou, H. 2016. Heavy metals in soils from a typical county in Shanxi Province, China: Levels, sources and spatial distribution. Chemosphere 148: 248-254. https://doi.org/10.1016/j. chemosphere.2015.12.049.

Roca, N., Pazos, M. y Bech, J. 2007. Disponibilidad de cobre, hierro, manganeso, zinc en suelos del $\mathrm{NO}$ Argentino. Ciencias del suelo 25(1):18502067.

Romero, K. 2009. Contaminación por metales pesados. Rev Cient Cienc Méd 12 (1):4546.

Sahuquillo, A., Rigol, A. and Rauret, G. 2003. Overview of the use of Leaching Extraction Tests for Risk Assessment of Trace Metals in Contaminated Soils and Sediments. Trends in Analytical Chemistry 22(3):152-159. https://doi.org/10.1016/ S0165-9936(03)00303-0.

Stankovic S, and Stankovic A. 2013. Bioindicators of Toxic Metals. En: Lichtfouse, E., Schwarzbauer,J. y Robert, D. (Ed). Green Materials for Energy, Products and Depollution. Springer. 3:151-228. https://doi.org/10.1007/97894-007-6836-9_5.

Seoánez, M. 1999. Contaminación del suelo: Estudios, tratamiento y gestión. Ediciones Mundi-Prensa, Madrid-BarcelonaMéxico, p147-149.

Wang, S., Wang, Y., Zhang, R., Wang, W., Xu, D., Guo, J., Li, P. and Yu, K. 2015. Historical levels of heavy metals reconstructed from sedimentary record in the Hejiang River, located in a typical mining region of Southern China. Science of the Total Environment 532: 645-654. https://doi. org/10.1016/j.scitotenv.2015.06.035. 
Silva, V., Lopes, S. and Gomes, E. 2014. Geochemistry and behavior of REE in stream sediments close to an old Sn-W mine, Ribeira, Northeast Portugal. Chemie der Erde 74(4): 545-555. https:// doi.org/10.1016/j.chemer.2013.08.002.

Sippl, K. 2015. Private and civil society governors of mercury pollution from artisanal and small-scale gold mining: A network analytic approach. The Extractive Industries and Society 2:198-208. https:// doi.org/10.1016/j.exis.2015.01.008.

Soil Survey Staff. 2010. Claves para la Taxonomía de Suelos. $11^{\mathrm{a}}$ Edicion. USDA- NRSC.

Vázquez, M., Poschenrieder, C. and Barcelo, J. 1989. Pulvinus structure arid leaí abscission in cadmium-treated bean plants (Phaseolus vulgaris). Canadian Journal of Botany 67(9): 2756-2764. https://doi.org/10.1139/b89-355.

Violantel, A., Cozzolino, V., Perelomov, L., Caporale, A. and Pigna, M. 2010. Mobility and bioavailability of heavy metals and metalloids in soil environments. Journal of Soil Science Plant Nutrition 10(3):268292. https://doi.org/10.4067/S071895162010000100005.
Yacoub, C., Pérez, F. and Miralles, N. 2012. Trace Metal Content of Sediments Close to Mine Sites in the Andean Region. The Scientific World Journal https://doi. org/10.1100/2012/732519.

Yin, H., Tan, N., Liu, C., Wang, J., Liang, X., Qu, M., Feng, X., Qiu, G., Tan,W. and Liu, F. 2016. The associations of heavy metals with crystalline iron oxides in the polluted soils around the mining areas in Guangdong Province, China. Chemosphere 161:181-189.

Zeng, F., Ali, S., Zhang, H., Ouyang, Y., Qiu, B., Wu, F. and Zhang, G. 2011. The influence of $\mathrm{pH}$ and organic matter content in paddy soil on heavy metal availability and their uptake by rice plants. Environmental Pollution 159 (1):84-91. https://doi. org/10.1016/j.envpol.2010.09.019. 